\title{
Influence of Laser-Drive Parameters on Annular Fast Electron Transport in Silicon
}

\author{
D. A. MacLellan ${ }^{1}$, D. C. Carroll², R. J. Gray ${ }^{1}$, A. P. L. Robinson ${ }^{2}$, M. P. \\ Desjarlais $^{3}$, D. Neely ${ }^{2}$, and P. McKenna ${ }^{1}$ \\ ${ }^{1}$ Department of Physics, SUPA, University of Strathclyde, Glasgow G4 0NG, UK \\ ${ }^{2}$ Central Laser Facility, STFC Rutherford Appleton Laboratory, Oxfordshire \\ OX11 0QX, UK \\ ${ }^{3}$ Sandia National Laboratories, P.O. Box 5800, Albuquerque, New Mexico 87185, \\ USA \\ December 20, 2013 \\ Corresponding author: Paul McKenna, Department of Physics, SUPA, \\ University of Strathclyde, Glasgow G4 0NG, United Kingdom. E-mail: \\ paul.mckenna@strath.ac.uk
}




\begin{abstract}
Three dimensional hybrid particle-in-cell (PIC) simulations are used to investigate the sensitivity of annular fast electron transport patterns in silicon to the properties of the drive laser pulse. It is found that the annular transport, which is induced by self-generated resistive magnetic fields, is particularly sensitive to the peak laser pulse intensity. The radius of the annular fast electron distribution can be varied by changing the drive laser pulse properties, and in particular the focal spot size. An ability to optically 'tune' the properties of an annular fast electron transport pattern could have important implications for the development of advanced ignition schemes and for tailoring the properties of beams of laser-accelerated ions.
\end{abstract}




\section{Introduction}

The large currents of relativistic (fast) electrons generated in the interaction of an ultraintense laser pulse with a solid can be used to drive the formation of strong fields and for heating applications, often at significant distances (hundreds of microns) from the source. Understanding the transport physics of these electrons in solids is therefore important for applications such as the fast ignition approach to inertial confinement fusion [1] and for tailoring the properties of beams of sheath-accelerated ions [2].

In recent years, attention has focused on the role of target electric resistivity in defining the properties of fast electron transport, both in terms of the different transport patterns in metals and insulators (see for example $[3,4,5]$ ), and in the possible use of self-generated resistive magnetic fields to control fast electron transport. As the fast electron population is accelerated into the target it draws a balancing cold return current, spatially overlapped with it and moving in the opposite direction, to satisfy the overall charge neutrality requirement [6]. If the fast electron current density is designated as $\mathbf{j}_{f}$ and the cold return current density as $\mathbf{j}_{r}$ then $\mathbf{j}_{f}+\mathbf{j}_{r} \approx 0$ [7]. From Ohm's law, the self-generated electric field is $\mathbf{E}=-\eta \mathbf{j}_{f}$, which when combined with Faradays law enables the self-generated magnetic field $\mathbf{B}$ to be written as $\frac{\partial \mathbf{B}}{\partial t}=$ $\eta \nabla \times \mathbf{j}_{f}+\nabla \eta \times \mathbf{j}_{f}$ where $\eta$ is the target resistivity and $t$ is time. The first term on the right hand side of this equation $\left(\eta \nabla \times \mathbf{j}_{f}\right)$ generates a magnetic field which pushes fast electrons into regions of higher current density, while the action of the second term $\left(\nabla \eta \times \mathbf{j}_{f}\right)$ is to push fast electrons into regions of higher resistivity. The resulting magnetic field strength and growth rate can be significant. Growth rates of $\sim 10^{14} \mathrm{Ts}^{-1}$ are produced in typical intense laser-solid interactions i.e. approximately $200 \mathrm{~T}$ in $1 \mathrm{ps}$. Fields of this magnitude have a strong effect on the propagation of multi- $\mathrm{MeV}$ electrons, and give rise to effects such as beam pinching or collimation [8], hollowing [9] and resistive instability-driven filamentation [10].

Schemes involving structured targets have been explored both experimentally $[11,12]$ and numerically [13] in an effort to use resistive gradients formed at the boundary between two different materials to induce magnetic field growth within solids, with the aim of collimating the fast electron beam. An extension of this layered target approach has been proposed to produce a 'magnetic switch-yard' scheme for controlling fast electron transport in advanced fusion targets [14]. In other work a technique for optically controlling the divergence of the electron beam by using double laser pulse drive has been demonstrated [15].

Recently we have shown that the resistivity of the solid at relatively low temperatures (eV to tens of eV regime) can have a defining influence on the properties of fast electron transport $[16,17]$. Using different forms (allotropes) of carbon, the influence of lattice structure in defining 
the resistivity evolution of the target and the subsequent onset of resistive fast electron beam filamentation was demonstrated [16]. It was shown that the shape of the resistivity-temperature profile of the target material plays a key role in defining the beam transport pattern. An annular beam profile was demonstrated in silicon, arising due to a dip in resistivity at a few $\mathrm{eV}$ [17]. The ability to fundamentally change the beam transport pattern from a Gaussian-like to an annular fast electron beam profile by controlling the resistively generated magnetic fields could have important consequences for applications. Alfvén showed that compared to a uniform beam, a higher current can be transported in an annular beam [18]. The Alfvén limit increases by a factor of $r / \delta r$, where $r$ is the radius of the beam and $\delta r$ is the width of the annulus. Davies [19] developed the idea by proposing schemes directly applicable to fast ignition, and Hatchett et al [20] discusses the advantages of using annular beams to reduce the energy requirements of the ignition drive beam. Annular fast electron beam transport in a solid can also be used to produce an annular beam of sheath-accelerated ions [17]. Temporal et al [21] proposes the use of an annular proton beam in combination with a second uniform proton beam to reduce the total energy required for ignition by a factor of almost two compared to a single uniform proton beam. Thus understanding and controlling the formation and subsequent evolution of annular fast electron beam transport is not only of fundamental interest, but may also have important implications for applications of intense laser-solid interactions.

In this paper, the sensitivity of annular fast electron beam transport to the parameters of the drive laser pulse is investigated numerically via 3D hybrid-PIC simulations. Changes to the size of the ring and the density of the electrons forming it are quantified as a function of laser pulse energy, duration and focal spot radius, and explained in terms of the effects on the resistive magnetic fields.

\section{Modelling}

The particle-based 3D-hybrid code ZEPHYROS $[11,14]$, in which the fast electrons are described using a PIC algorithm while the background plasma is treated as a fluid [22, 23], is used. A 200 $\mu \mathrm{m} \times 400 \mu \mathrm{m} \times 400 \mu \mathrm{m}$ simulation grid was used, with a cell resolution of $\Delta \mathrm{X}=\Delta \mathrm{Y}=\Delta \mathrm{Z}$ $=1 \mu \mathrm{m}$. As with many other hybrid-PIC codes, the fast electron source is not calculated selfconsistently. The properties of the electron beam at the source are defined based on previous theory, experiment and PIC simulation studies of fast electron generation, and the electrons are introduced or injected into the computational grid in a 'laser-spot' profile region. The electron source is centred at $[\mathrm{X}, \mathrm{Y}, \mathrm{Z}]=[0,0,0]$, with a transverse intensity profile determined by $I=\alpha I_{L} \exp \left(-\frac{r}{r_{L}}\right)$, where $r$ and $r_{L}$ are the radius and focal spot radius respectively, $I_{L}$ 
is the peak laser intensity and $\alpha$ is the fraction of laser energy converted to fast electrons (set to 0.3). The electron pulse has a top-hat temporal profile. The fast electrons propagate in the X-direction with an exponential energy distribution (of the form $\exp \left(-\frac{E}{T_{f}}\right)$ ), where $E$ is the electron kinetic energy and the mean temperature $T_{f}$ scales ponderomotively $\left(k_{B} T_{f}=\right.$ $0.511\left[\left(1+0.73 I_{18} \lambda_{\mu m}^{2}\right)^{1 / 2}-1\right] \mathrm{MeV}$, where $I_{18}$ is the peak laser intensity in units of $10^{18} \mathrm{Wcm}^{-2}$ and $\lambda_{\mu m}$ is the laser wavelength in microns [24]). The electrons are injected with a uniform angular distribution over a cone subtended by a half-angle of $50^{\circ}$ [25] and in all cases the initial target temperature is set equal to $1 \mathrm{eV}$.

Although the fast electron source parameters, as defined by the percentage of the laser energy absorbed by the electrons and their injection angle and distribution, are expected to vary with the properties of the laser pulse, the precise nature of these dependencies are still not well understood and are the subject of ongoing work. Therefore, as this investigation focuses on the transport pattern of the fast electron beam within the target, the source properties are kept constant throughout.

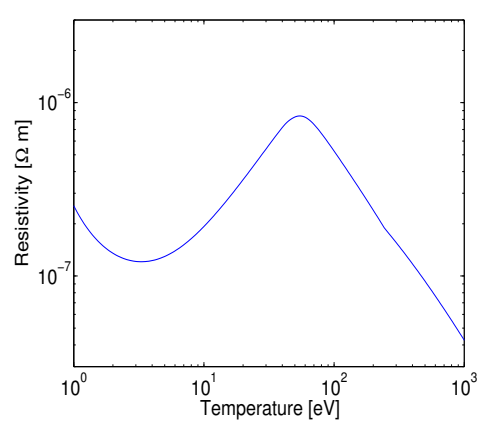

(a)

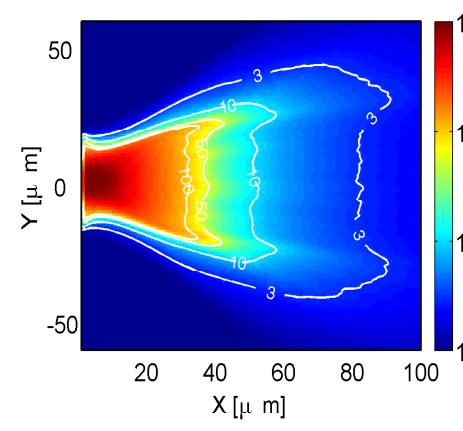

(b)

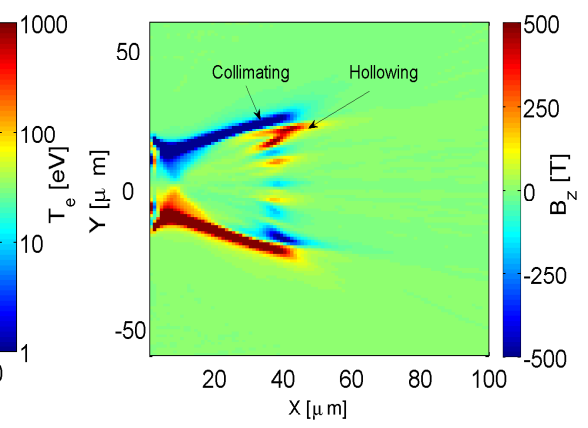

(c)

Figure 1: (a) Electrical resistivity of silicon as a function of target temperature, based on ab initio QMD calculations coupled with the Kubo-Greenwood equation [17]. (b, c) Example hybrid-PIC simulation results showing $2 \mathrm{D}$ maps (1400 fs into the simulation) of (b) the target temperature in $\mathrm{eV}$, with selected isothermal contours, and (c) the corresponding magnetic flux density $\left(B_{Z}\right.$ component in Tesla), showing a collimating component which acts to limit the divergence of the beam and a hollowing component arising from a reversal in the magnetic field direction inside the edge of the beam.

The silicon resistivity-temperature profile used, presented in figure 1(a), is based on a quantum molecular dynamics (QMD) Kubo-Greenwood calculation [26, 27], as discussed in MacLellan et al [17]. In that work, it was shown that the dip in the resistivity at a few eV leads to a reversal 
in the resistivity gradient near the edges of the fast electron beam, where the target is heated to relatively low temperatures. An example 2D temperature map from the simulations (for $I_{L}$ $=5 \times 10^{20} \mathrm{Wcm}^{-2} ; E_{L}=192 \mathrm{~J} ; \tau_{L}=1 \mathrm{ps}$ and $\left.r_{L}=3.5 \mu \mathrm{m}\right)$ is shown in figure $1(\mathrm{~b})$ and the corresponding 2D map of magnetic flux density is shown in 1(c), (both sampled after $1400 \mathrm{fs}$ ). This reversal in the resistivity gradient leads to a reversal in the direction of the self-generated resistive magnetic field, via the $\nabla \eta \times \mathbf{j}_{f}$ term. The combination of the collimating effect of the azimuthal magnetic field enveloping the beam (the collimating component) and the reversed magnetic field just inside the edge of the beam (the hollowing component) drive a localized increase in the fast electron beam current density in an annular profile. If the ring is of sufficient size and contains a large enough fraction of the total electron current, the transport pattern is maintained and even reinforced as the electrons propagate across the remainder of the target. The reinforcement occurs due to the local increase in resistive heating arising from the increase in $\mathbf{j}_{f}$ (resistive heating scales as $\mathbf{j}_{f}^{2}$ ) which drives a local increase in resistivity and thus larger resistivity gradients and azimuthal B-fields surrounding the annulus.

\section{Simulation Results}

We begin by defining two parameters to quantify annular transport in the simulation results. These are: (1) the inner radius of the annulus at the end of the simulation box, i.e. at $\mathrm{X}=$ $200 \mu m$ (this is defined as the ratio at which the electron density increases by a factor of 5 with respect to the axial density); and, (2) the ratio of the electron densities in the annulus to the axial position $([200,0,0])$, again at the end of the simulation box. These quantities effectively measure the size of the ring and the annulus-to-axial contrast ratio.

To investigate the sensitivity of these parameters to the drive laser pulse parameters, three series of simulation scans were performed, as follows: A) variation of laser pulse energy, $E_{L}$ in the range $78-385 \mathrm{~J}$, for fixed focal spot radius $r_{L}=3.5 \mu \mathrm{m}$ and fixed laser pulse duration $\tau_{L}=$ $1 \mathrm{ps}$; B) variation of $r_{L}$ in the range $2.5-5.5 \mu \mathrm{m}$, for fixed $E_{L}=192 \mathrm{~J}$ and $\tau_{L}=1 \mathrm{ps}$; and, C) variation of $\tau_{L}$ in the range $0.5-2.5 \mathrm{ps}$, for fixed $E_{L}=192 \mathrm{~J}$ and $r_{L}=3.5 \mu \mathrm{m}$. These parameter

ranges were chosen such that the peak laser pulse intensity, $I_{L}$, was varied in the range $2 \times 10^{20}$ $-1 \times 10^{21} \mathrm{Wcm}^{-2}$ for all three scans.

Figure 2 presents the results from these simulations. The fast electron density in the $[\mathrm{X}$, Y] mid-plane is shown, at an example time of $\tau_{L}+0.4 \mathrm{ps}-$ this time is chosen such that the full population of electrons has been initiated in the simulation and has propagated at least 100 $\mu \mathrm{m}$ across the target, the majority having reached the rear surface. We find that the stability of the annular transport pattern is maintained until significant refluxing of electrons, driven 
by reflection at the target rear side, occurs in the simulation (fast electron refluxing has been previously shown to perturb the resistive magnetic field structure within solids [28]). Scan A is presented in the top row (figure $2(\mathrm{a}-\mathrm{e})$ ), B in the middle row (figure $2(\mathrm{f}-\mathrm{j})$ ), and $\mathrm{C}$ in the bottom row (figure $2(\mathrm{k}-\mathrm{o})$ ). The corresponding simulation outputs for the $[\mathrm{Y}, \mathrm{Z}]$ rear-plane snapshots are displayed in figure 3 .

$2 \times 10^{20} \mathrm{Wcm}^{-2} \quad 2.5 \times 10^{20} \mathrm{Wcm}^{-2} \quad 3.3 \times 10^{20} \mathrm{Wcm}^{-2} 5 \times 10^{20} \mathrm{Wcm}^{-2} \quad 1 \times 10^{21} \mathrm{Wcm}^{-2}$

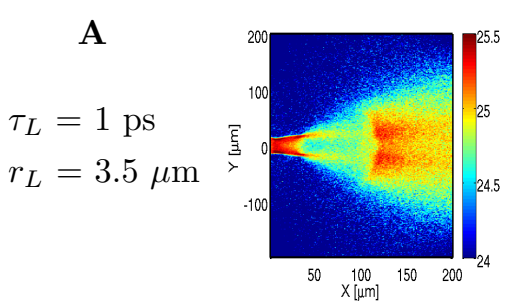

(a) $E_{L}=78 \mathrm{~J}$

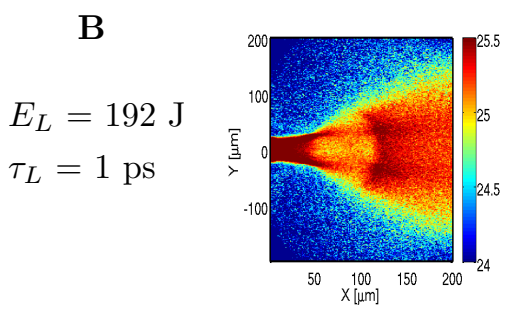

(f) $r_{L}=5.5 \mu \mathrm{m}$

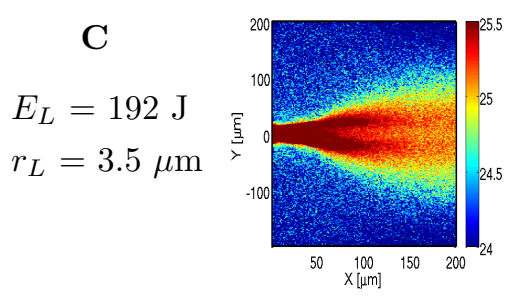

(k) $\tau_{L}=2.5 \mathrm{ps}$

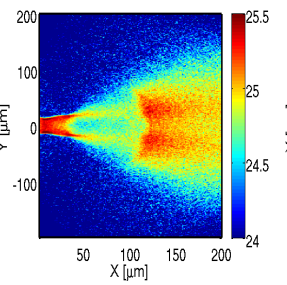

(b) $E_{L}=96 \mathrm{~J}$

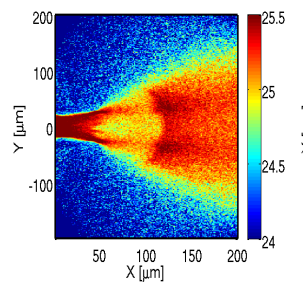

(g) $r_{L}=5 \mu \mathrm{m}$

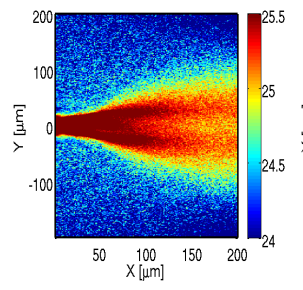

(l) $\tau_{L}=2 \mathrm{ps}$

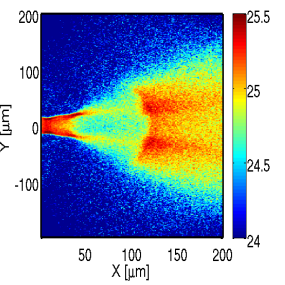

(c) $E_{L}=128 \mathrm{~J}$

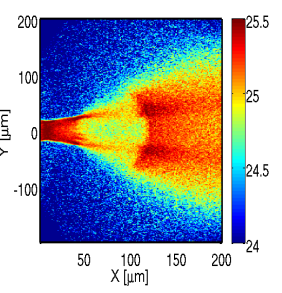

(h) $r_{L}=4.3 \mu \mathrm{m}$

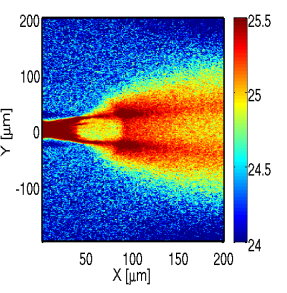

(m) $\tau_{L}=1.5 \mathrm{ps}$

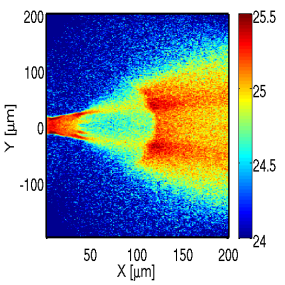

(d) $E_{L}=192 \mathrm{~J}$

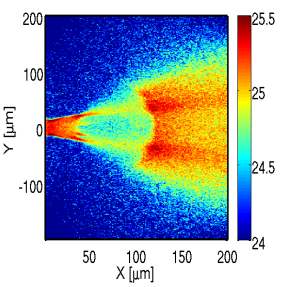

(i) $r_{L}=3.5 \mu \mathrm{m}$

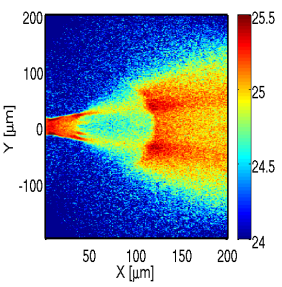

(n) $\tau_{L}=1 \mathrm{ps}$

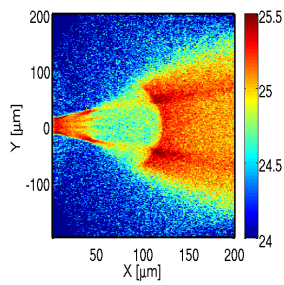

(e) $E_{L}=384 \mathrm{~J}$

Figure 2: $2 \mathrm{D}$ maps of the fast electron beam density $\left(\log _{10}\right)$, in units of $\mathrm{m}^{-3}$, in the $[\mathrm{X}-\mathrm{Y}]$ mid-plane of the simulation, for three laser pulse parameter scans: (a-e) variation of $E_{L}$ (top row); (f-j) variation of $r_{L}$ (middle row); (k-o) variation of $\tau_{L}$ (bottom row). The value of the varied parameter is given below each panel. 

$2 \times 10^{20} \mathrm{Wcm}^{-2}$
$2.5 \times 10^{20} \mathrm{Wcm}^{-2}$
$3.3 \times 10^{20} \mathrm{Wcm}^{-2}$
$5 \times 10^{20} \mathrm{Wcm}^{-2}$
$1 \times 10^{21} \mathrm{Wcm}^{-2}$

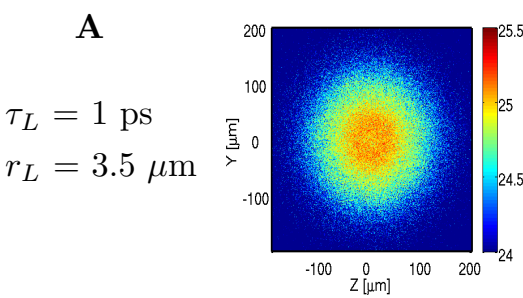

(a) $E_{L}=78 \mathrm{~J}$

B

$E_{L}=192 \mathrm{~J}$

$\tau_{L}=1 \mathrm{ps}$

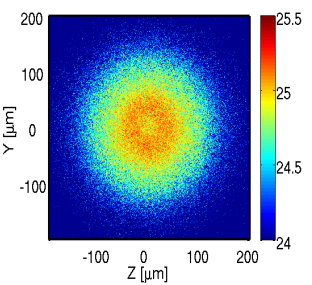

(b) $E_{L}=96 \mathrm{~J}$

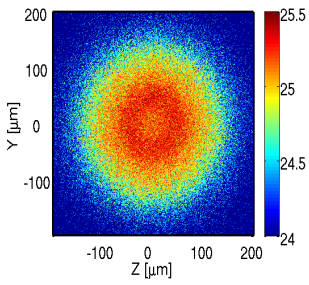

(g) $r_{L}=5 \mu \mathrm{m}$

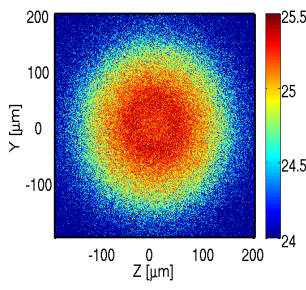

(f) $r_{L}=5.5 \mu \mathrm{m}$

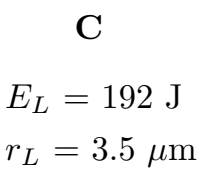

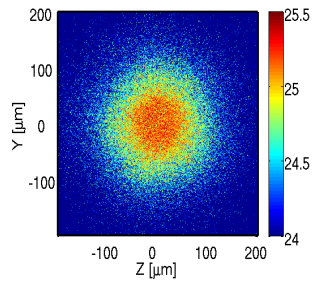

(k) $\tau_{L}=2.5 \mathrm{ps}$

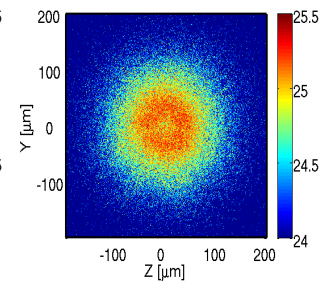

(l) $\tau_{L}=2 \mathrm{ps}$

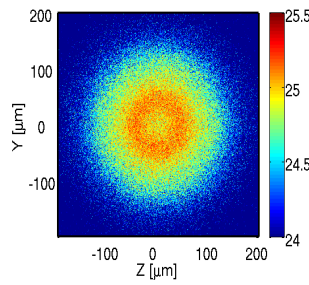

(c) $E_{L}=128 \mathrm{~J}$

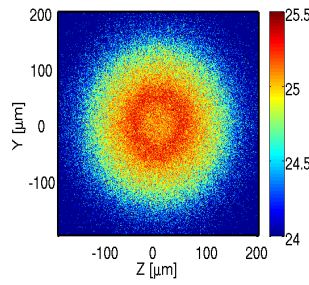

(h) $r_{L}=4.3 \mu \mathrm{m}$

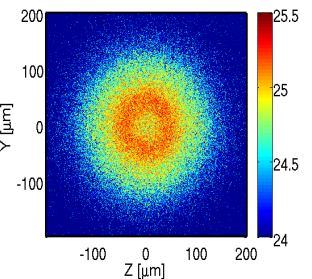

(m) $\tau_{L}=1.5 \mathrm{ps}$

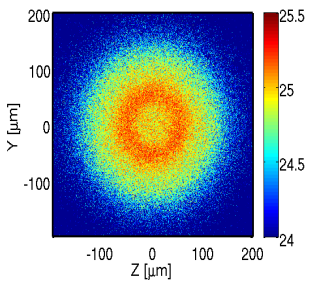

(d) $E_{L}=192 \mathrm{~J}$

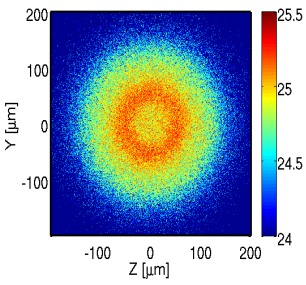

(i) $r_{L}=3.5 \mu \mathrm{m}$

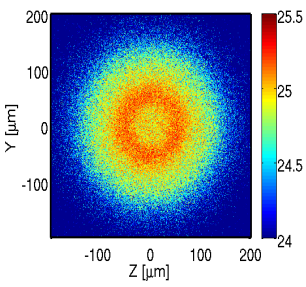

(n) $\tau_{L}=1 \mathrm{ps}$

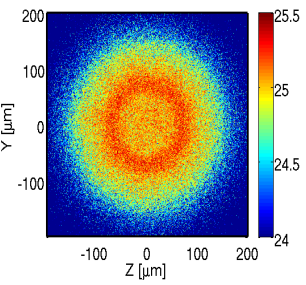

(e) $E_{L}=385 \mathrm{~J}$

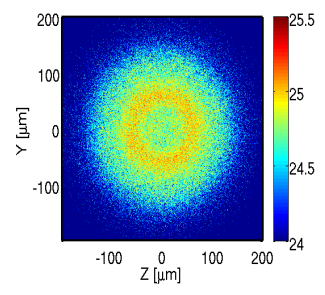

(j) $r_{L}=2.5 \mu \mathrm{m}$

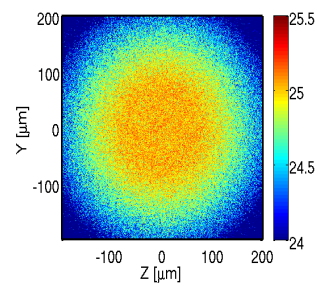

(o) $\tau_{L}=0.5 \mathrm{ps}$

Figure 3: Same as figure 2, but for the rear surface [Y-Z] plane

In scan $\mathrm{A}$, we find that increasing the energy from $78 \mathrm{~J}$ to $385 \mathrm{~J}$, which effectively increases the current of electrons, results in an increase in the overall divergence of the electron beam and thus an increase in the radius of the annular feature. By contrast, when increasing the intensity over the same range by decreasing the focal spot radius from $r_{L}=5.54 \mu \mathrm{m}$ to $2.48 \mu \mathrm{m}$, scan B, the increase in both the overall beam divergence and the radius of the ring is smaller. Note that the fixed laser pulse energy in combination with the increasing laser intensity means that fewer electrons are injected as the focal spot radius is decreased, producing the beam density decrease observed in figure $2(\mathrm{f}-\mathrm{j})$. This influences the target temperature and resistivity evolution, and subsequent electron transport properties, as will be explained later. In scan C, significant changes in the beam transport are found for variation of the pulse duration. As $\tau_{L}$ is increased over the range $1 \mathrm{ps}$ to $2.5 \mathrm{ps}$, the overall beam divergence and radius of the annular structure are found to decrease, effectively leading to more uniform beam transport for the longer pulse durations (see figure $2(\mathrm{n}-\mathrm{k})$. However, reducing $\tau_{L}$ to $0.5 \mathrm{ps}$ also results in a loss of the annular 
structure and a significant increase in the overall fast electron beam divergence which, as will be discussed later, is a result of a reduction in the magnetic field strength.

To examine these trends more closely, the rear-surface simulation outputs from figure 3 are analysed to quantify the variation of the size of the annulus and the relative density of electrons contained within it, as a function of $I_{L}$ for each of the three scans. The radius of the annular structure is defined as the distance from the centre of the rear-surface simulation grid (i.e. at [Y, $\mathrm{Z}]=[0,0])$ to the inner annulus of the ring structure, and the results are shown in figure 4(a). Note that the $10^{21} \mathrm{Wcm}^{-2}$ point in scan $\mathrm{C}\left(\tau_{L}=0.5 \mathrm{ps}\right)$ is excluded as a clear ring profile is not observed for this case. Figure 4(b) shows the ratio of the fast electron density in the annulus (peak density) to the density of the centre of the beam, $[\mathrm{Y}, \mathrm{Z}]=[0,0]$.

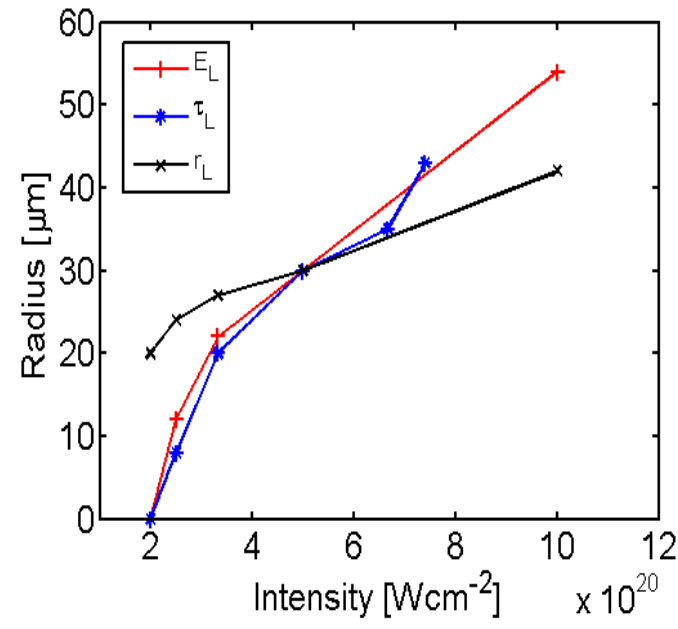

(a)

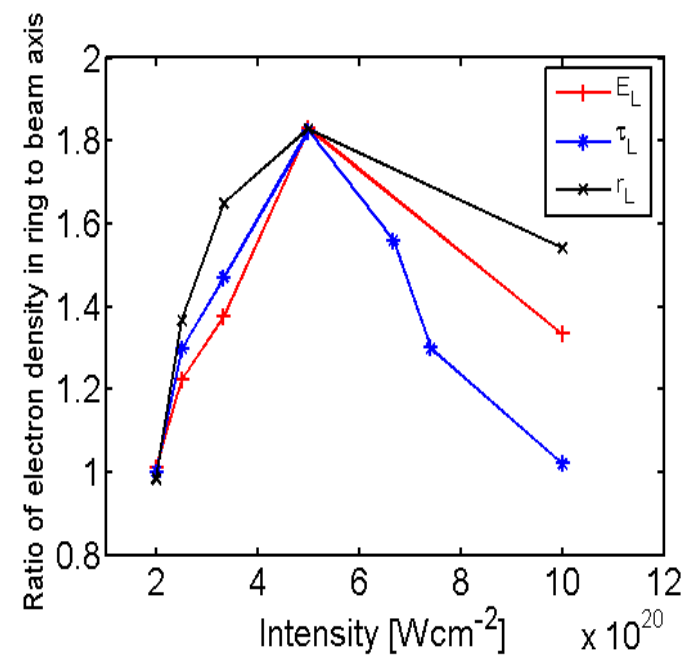

(b)

Figure 4: (a) Inner radius of the annulus at the target rear surface as a function of $I_{L}$, for the three parameter scans shown in figure 2 and figure 3. (b) Ratio of the fast electron density in the annulus to the density on-axis (i.e. at $[200,0,0]$ ) as a function of $I_{L}$, for the same three parameter scans.

\section{Overall effect of laser intensity}

Considering first the variation of the size of the ring, we find that the radius increases with increasing peak laser intensity for all three scans. This is due to the increase in the overall divergence of the fast electron beam with increasing $I_{L}$. Since the magnetic field reversal driving the hollowing effect occurs close to the edge of the beam (where target heating is of the order 
of a few eV), the radius of the ring is, to first order, defined by the overall beam divergence. The divergence in turn depends on the angular distribution of the electrons at the source (which is fixed in our simulations) and the strength of the collimating magnetic field. The field is strongest within the first few tens of microns from the front surface where the current density is highest and can be approximated as a cylinder - hollowing is not seeded until a depth of $\sim 40 \mu \mathrm{m}$ (as shown in figure 1(c)). The resistive azimuthal magnetic field around a uniform cylindrical electron beam of radius $r_{f}$ can be estimated as $\frac{\partial \mathbf{B}}{\partial t}=\eta \mathbf{j}_{f} / r_{f}$, where the fast electron current is given by $\mathbf{j}_{f}=I_{f} / T_{f}$, in which $I_{f}$ is the fast electron beam intensity [8]. The inverse dependence of the strength of the collimating magnetic field on the fast electron energy $T_{f}$ is due to the decrease in $\mathbf{j}_{f}$ as $T_{f}$ is increased. $T_{f}$ scales ponderomotively with the square root of the laser intensity, and hence as the peak laser intensity increases the magnetic field strength and therefore it's global pinching effect on the fast electron beam decreases, increasing the overall beam divergence. Thus the radius of the ring induced by the hollowing component of the field formed near the edge of the beam increases with $I_{L}$. We note that the laser-to-electron energy conversion efficiency is likely to change with $I_{L}$, which will also effect magnetic field growth. However the precise dependencies of the energy coupling efficiency on each of the laser pulse parameters are not well enough known to factor this into the study and therefore, as highlighted in section 2, this parameter is fixed.

As shown in figure 4(b), for all three pulse parameter scans the ratio of the electron density in the annulus to the axial density is highest for $I_{L}=5 \times 10^{20} \mathrm{Wcm}^{-2}$ (the same result at this intensity forms part of the simulation set for all three scans). With decreasing laser intensity, the overall beam divergence and ring radius decreases (due largely to the reduction in $T_{f}$, as discussed above) resulting in a higher beam density on-axis. Although hollowing still occurs at low $I_{L}$, the ratio of the beam densities in the annulus to the axial position decreases as the collimating effect of the magnetic field dominates over the hollowing term for small beam radii. With increasing $I_{L}$ above $5 \times 10^{20} \mathrm{Wcm}^{-2}$, the rate of the resistive magnetic field growth decreases. Therefore, although the beam radius increases, which should enhance the ratio, the lower magnitude of the hollowing magnetic field and higher $T_{f}$ results in less electrons being deflected to form the ring. Since the local $\mathbf{j}_{f}$ of the annulus thereby decreases, the rate of localised resistive heating is lower and so the reinforcing feedback condition discussed above is not established. The annular transport structure does not develop properly in this case. 


\section{Laser focal spot dependence}

Although for all three parameter scans, the ring radius increases with $I_{L}$, it is clear from figure 4 (a) that the scaling with intensity is different when varying the focal spot radius compared to variation of either $E_{L}$ or $\tau_{L}$. This difference is accounted for by the size of the electron beam at source. A decrease in $r_{L}$ has two main effects: (1) $\mathbf{j}_{f}$, and hence $\mathbf{B}$, increases, decreasing the overall divergence of the beam; and, (2) the radius at which hollowing is seeded decreases. Both effects influence the radius of the beam annulus downstream, and we consider each in turn:

(1) The magnetic field which defines the overall beam divergence scales inversely with $r_{f}$, as discussed above, and is strongest at the source, where $\mathbf{j}_{f}$ is highest and defined by the size of the laser focal spot. Let us consider the simulation result at $5 \times 10^{20} \mathrm{Wcm}^{-2}$, which is common to all three scans, and for which $r_{L}=3.5 \mu \mathrm{m}$, as a reference. As $r_{L}$ is decreased to $2.5 \mu \mathrm{m}$ $\left(1 \times 10^{21} \mathrm{Wcm}^{-2}\right), \mathbf{j}_{f}$ at the source, and hence the peak magnetic field strength, is enhanced by approximately a factor of two. The simulation result shows a reduction in the annulus radius, corresponding to an overall beam divergence angle reduction of $\sim 36 \%$, compared to the simulations at the same intensity in scans A and C for which $r_{L}=3.5 \mu \mathrm{m}$. Similarly, an increase in $r_{L}$ from $3.5 \mu \mathrm{m}$ to $4.3 \mu \mathrm{m}\left(I_{L}=3.3 \times 10^{20} \mathrm{Wcm}^{-2}\right)$ results in a $50 \%$ decrease in $\mathbf{j}_{f}$ and $\mathbf{B}$ at the source, which contributes to the $\sim 35 \%$ increase in the ring divergence in the simulation results (again compared to the simulations at the same intensity in scans $\mathrm{A}$ and $\mathrm{C}$ ).

(2) The effect of varying $r_{L}$ on the radius at which the annular transport pattern is seeded is explored in figure 5. The transverse intensity profiles of the drive laser pulse are shown in figure 5(a) and (d) for the energy and focal spot scans (A and B), respectively. For brevity, scan $\mathrm{C}$ is not considered in detail because the results are very similar to A. For each scan, three example $I_{L}$ are considered: $2 \times 10^{20} \mathrm{Wcm}^{-2}, 5 \times 10^{20} \mathrm{Wcm}^{-2}$ and $1 \times 10^{21} \mathrm{Wcm}^{-2}$ (displayed as the black, red and blue plots, respectively) and the full width at half maximum (FWHM) for each is marked. The corresponding target temperature and resulting resistivity profiles (along the $\mathrm{Y}$-axis) at $\mathrm{X}=50 \mu \mathrm{m}$ (depth at which the annular transport evolves) are shown in figure 5(b) - (c) and figure 5(e) - (f) for scan A and B respectively. The red dotted line highlights the temperature at $3.5 \mathrm{eV}$ and the corresponding reversal in the resistivity gradient at the edges of the beam which seeds annular transport [17]. From these plots we see that the radius at which seeding of the annular transport occurs increases with intensity for scan A (and C), but decreases slightly with intensity for scan B, due to the change in $r_{L}$. For $I_{L}=2 \times 10^{20}$ $\mathrm{Wcm}^{-2}$, for example, the radius at which annular transport is seeded is $\sim 35 \%$ larger in scan $\mathrm{B}$ than A, due to the larger $r_{L}$, which contributes to the larger annular profile at the rear of the simulation box. 


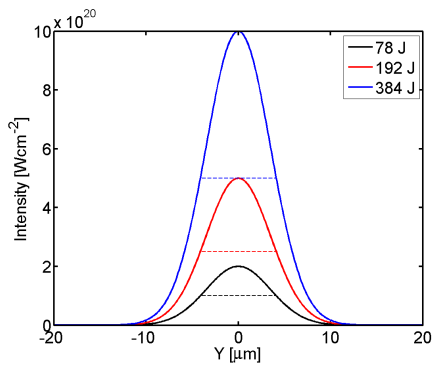

(a) Intensity (varying $E_{L}$ )

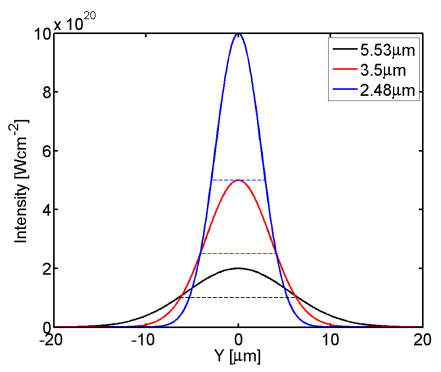

(d) Intensity (varying $r_{L}$ )

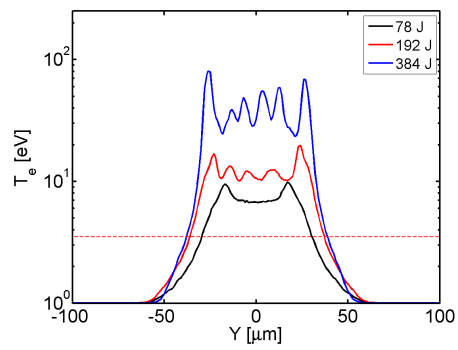

(b) Temperature (varying $E_{L}$ )

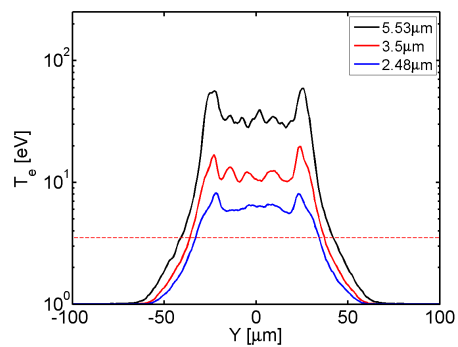

(e) Temperature (varying $r_{L}$ )

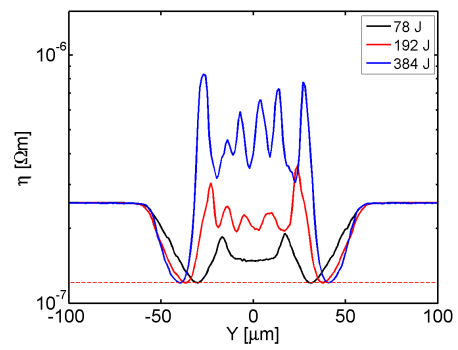

(c) Resistivity (varying $E_{L}$ )

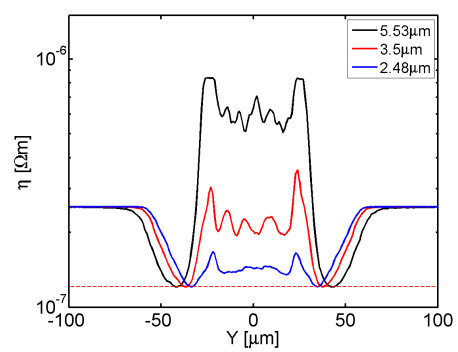

(f) Resistivity (varying $r_{L}$ )

Figure 5: Variation of (a) the laser pulse intensity, (b) the target temperature at $\mathrm{X}=50 \mu \mathrm{m}$ and (c) electrical resistivity at $\mathrm{X}=50 \mu \mathrm{m}$ along the $\mathrm{Y}$-axis, all for three example peak intensities $\left(2 \times 10^{20} \mathrm{Wcm}^{-2}\right.$-black, $5 \times 10^{20} \mathrm{Wcm}^{-2}$-red and $1 \times 10^{21} \mathrm{Wcm}^{-2}$-blue $)$ obtained by variation of $E_{L}$ (values given). (d-f) Same as (a-c), for the same three peak intensities, but for variation of $r_{L}$ (values given). Dotted lines in (a) and (c) highlight the FWHM of the intensity distributions. Dotted lines in (b) and (e) mark the important $3.5 \mathrm{eV}$ target temperature, and in (c) and (f) mark the corresponding turning points in the target resistivity which seeds annular fast electron beam transport.

Note that the simulation profiles in figure 5 show spikes in target temperature which grow at the edge of the beam and arise due to the local increase in the beam density (and hence collisional return current) due to the deflection induced by the magnetic field. As discussed above and in MacLellan et al [17], this drives a localized increase in resistivity for temperatures above $\sim 3.5 \mathrm{eV}$ and hence the region near the edge of the beam remains more resistive than the centre, which reinforces the annular transport pattern as the beam propagates over the remainder of the target.

With reference to figure $5(\mathrm{e})$, it is interesting to note that reducing $r_{L}$ for fixed $E_{L}$ and $\tau_{L}$ significantly reduces the overall heating of the target. This occurs because $I_{L}$ and hence $T_{f}$ increases, which for a fixed total fast electron energy (assuming a fixed energy conversion 
efficiency from laser to electrons), reduces the total number of fast electrons, and hence $\mathbf{j}_{f}^{2}$ heating. Additionally, we note that there is a general trend of the onset of fast electron beam filamentation with increasing $E_{L}$, as manifested in the temperature oscillations across the beam observed in 5(b). This is a consequence of the increase in background temperature between $\sim 3.5 \mathrm{eV}$ and $\sim 70 \mathrm{eV}$, which produces a high resistivity (figure 1), leading to a higher resistive instability growth rate [16].

\section{Influence of laser pulse duration}

To explore why the annular transport pattern is washed out for the $\tau_{L}=0.5$ ps simulation in scan $\mathrm{C}$, the spatial profile of the resistively generated magnetic field is examined as a function of $\tau_{L}$. Lineouts are extracted along the Y-direction (i.e. transverse, or radial, profile) of the simulation grid, at the $\mathrm{X}$-axis position to which the magnetic field extends (which changes with $\left.\tau_{L}\right)$. This is defined as the position at which the magnitude of the collimating magnetic field is reduced by $50 \%$. For the cases shown in figure $6(\mathrm{a})$, this represents depths of $45 \mu \mathrm{m}, 50 \mu \mathrm{m}, 55$ $\mu \mathrm{m}, 60 \mu \mathrm{m}$ and $65 \mu \mathrm{m}$ for $\tau_{L}=0.5 \mathrm{ps}, 1 \mathrm{ps}, 1.5 \mathrm{ps}, 2 \mathrm{ps}$ and $2.5 \mathrm{ps}$ respectively. The resulting field profiles are shown in figure 6 (a) for radius up to $80 \mu \mathrm{m}$ (i.e. $\mathrm{Y}=0$ corresponds to the centre of the beam) for each of the five simulations. These plots demonstrate the complex interplay between pulse duration and the generation of the collimating magnetic field which envelopes the beam, forcing the electrons towards the axis (positive $B_{Z}$ in figure 6(a)), and the hollowing reversed magnetic field which forms just inside the circumference of the beam, driving electrons outwards (negative $B_{Z}$ in figure 6(a)). Achieving a balance between these opposing azimuthal components of the magnetic field is important for seeding the annular transport pattern. 


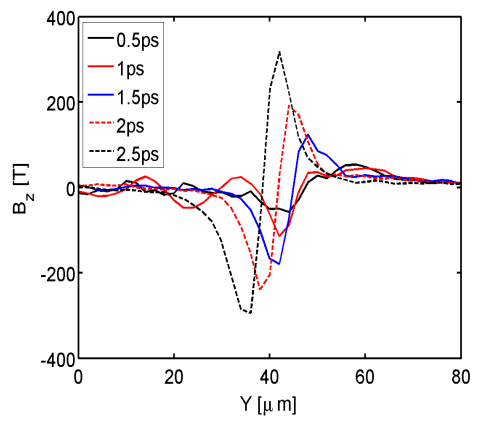

(a)

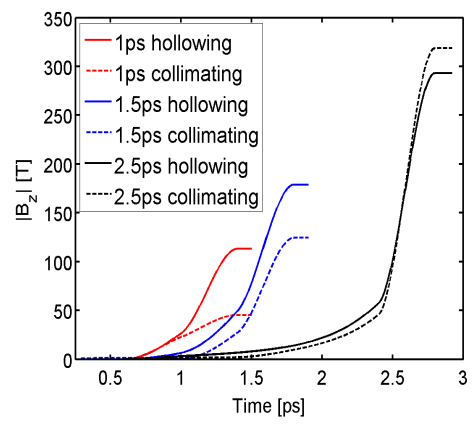

(b)

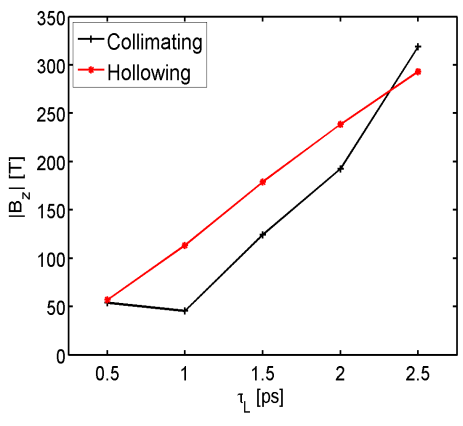

(c)

Figure 6: (a) Variation of the self-generated resistive magnetic field as a function of beam radius (Y-axis) at the penetration depth over which the magnetic field extends: $45 \mu \mathrm{m}, 50 \mu \mathrm{m}, 55 \mu \mathrm{m}$, $60 \mu \mathrm{m}$ and $65 \mu \mathrm{m}$ for $\tau_{L}=0.5 \mathrm{ps}, 1 \mathrm{ps}, 1.5 \mathrm{ps}, 2 \mathrm{ps}$ and $2.5 \mathrm{ps}$ respectively. (b) Magnitude of the collimating and hollowing magnetic field components as a function of time for given $\tau_{L}$. (c)

Magnitude of the collimating and hollowing magnetic field components as a function of $\tau_{L}$.

The magnitude and rate of the resistive magnetic field growth depend on both the fast electron current density and bunch duration (effectively the drive laser-pulse duration) [6]. Figure 6(a) shows example profiles of the magnetic field, exhibiting both the collimating component and the oppositely-directed hollowing component, for given $\tau_{L}$. We find that the amplitude of both magnetic field components increases with pulse duration, as a consequence of the longer duration over which the field grows [22], and generally that the differences between the magnitude of the components decreases. This is observed in figure 6(b) which shows the temporal evolution of both magnetic field components for three example $\tau_{L}$, and figure $6(\mathrm{c})$ showing the magnitude of both components as a function of pulse duration. The greatest difference between the two components is observed for $\tau_{L}=1 \mathrm{ps}$, which explains the resulting strong annular transport patterns. With increasing pulse duration the difference decreases, as the collimating field becomes more dominant. Eventually for $\tau_{L}$ above $2 \mathrm{ps}$, there is a switch over in the dominant field component. As a result, the annular transport pattern is no longer sustained and the rearsurface fast electron spatial profile becomes uniform, as seen in figure 3(k). Furthermore, for the shortest pulse duration explored (i.e. $\tau_{L}=0.5 \mathrm{ps}$ ), the peak collimating magnetic field is approximately balanced by the peak hollowing magnetic field, and the magnitude of each is not large enough to seed significant annular transport. In general, we find that the best conditions for seeding a strong annular transport pattern occurs when the magnitude of the hollowing component is larger than the collimating component. 
With reference to figure 6(a), we also find that with increasing pulse duration the position of the maximum of each field component in the Y-axis decreases (i.e. the radius of the ring decreases). This is a direct consequence of the increasing collimating field which acts to decrease the overall beam divergence.

\section{Conclusions}

In conclusion, the annular transport patterns of fast electron beams in silicon, arising from oppositely-directed azimuthal components of the self-generated resistive magnetic field, have been investigated as a function of the parameters of the drive laser pulse, using a 3D hybridPIC code. The results demonstrate that there is an optimum laser peak intensity range for transporting fast electrons within an annular structure produced in this way. The size of the annulus is found to increase with peak intensity, due to a decrease in the magnitude of the collimating magnetic field which defines the overall beam divergence. We find that there is an optimum laser intensity for enhancing the annulus-to-axial electron density contrast ratio, which is determined by the relative strength of the resistive magnetic field components. The hollowing component should be high to deflect electrons into the annulus, but the collimating component should not be so high as to produce a strongly collimated beam. The resulting trade-off explains the observed optimum laser drive intensity. We further find that the size of the annular profile is sensitive to the laser focal spot size, which enables some degree of tuning of the annular transport pattern for a fixed beam temperature or drive laser intensity.

\section{Acknowledgements}

We acknowledge computing resources provided by STFC's e-Science project. This work is financially supported by EPSRC (grant numbers EP/J003832/1, EP/L001357/1 and EP/K022415/1). The research leading to these results has also received funding from LASERLAB-EUROPE (grant agreement $n^{\circ}$ 284464, EC's Seventh Framework Programme) and is sponsored by the Air Force Office of Scientific Research, Air Force Material Command, USAF, under grant number FA8655-13-1-3008. The U.S Government is authorized to reproduce and distribute reprints for Governmental purpose notwithstanding any copyright notation thereon.

\section{References}

[1] M. Tabak et al., Phys. Plasmas 1, 1626 (1994). 
[2] M. Borghesi et al., Fusion Sci. Technol. 49, 412 (2006).

[3] J. Fuchs et al., Phys. Rev. Lett, 91, 255002 (2003).

[4] A. J. Kemp et al., Phys. Plasmas, 11, L69 (2004).

[5] M. N. Quinn et al., Plasma Phys. Control. Fusion 53, 124012 (2011).

[6] A. R. Bell et al., Plasma Phys. Control. Fusion., 48, R37 (2006).

[7] Y. Sentoku et al., Phys. Rev. Lett, 90, 155001 (2003).

[8] A. R. Bell and R. J. Kingham, Phys. Rev. Lett, 91, 035003 (2003).

[9] P. A. Norreys et al., Plasma Phys. Control. Fusion, 48, L11 (2006).

[10] L. Gremillet et al., Phys. Plasmas 9, 941 (2002).

[11] S. Kar et al., Phys. Rev. Lett. 102, 055001 (2009).

[12] B. Ramakrishna et al., Phys. Rev. Lett, 105, 135001 (2010).

[13] A. P. L. Robinson and M. Sherlock, Phys. Plasmas 14, 083105 (2007).

[14] A. P. L. Robinson et al., Phys. Rev. Lett. 108, 125004 (2012).

[15] R. H. H. Scott et al., Phys. Rev. Lett, 109, 015001 (2012).

[16] P. McKenna et al., Phys. Rev. Lett, 106, 185004 (2011).

[17] D. A. MacLellan et al., Phys. Rev. Lett, 111, 167588 (2013).

[18] H. Alfven, Phys. Rev. 55, 425 (1939).

[19] J. R. Davies, Phys. Rev. E, 69, 065402 (2004).

[20] S. P. Hatchett et al., Fusion Sci. Technol. 49, 327 (2006).

[21] M. Temporal et al., Phys. Plasmas, 15, 052702 (2008).

[22] J. R. Davies et al., Phys. Rev. E 56, 7193 (1997).

[23] J. R. Davies, Phys. Rev. E 65, 026407 (2002).

[24] S. C. Wilks and W. L. Kruer, IEEE J. Quantum Electron. 33, 1954 (1997).

[25] M. Coury et al., Phys. Plasmas 20, 043104 (2013). 
[26] M. P. Desjarlais, J. D. Kress, and L. A. Collins, Phys. Rev. E 66, 025401 (2002).

[27] G. Kresse and J. Hafner, Phys. Rev. B 47, 558 (1993).

[28] X. H. Yuan et al., New J. Phys. 12, 063018 (2010). 